\title{
Singularity image method for electrical impedance tomography of bubbly flows
}

\author{
Cunkui Huang ${ }^{1}$, Jinhee Lee ${ }^{2}$, William W Schultz ${ }^{3}$ and Steven L Ceccio ${ }^{3}$ \\ ${ }^{1}$ Institute of Engineering Thermophysics, Chinese Academy of Science, \\ People's Republic of China \\ 2 Department of Mechanical Information, Hongik University, Seoul, Korea \\ ${ }^{3}$ Department of Mechanical Engineering, University of Michigan, Ann Arbor, MI, USA \\ E-mail: CunkuiHuang@hotmail.com,jinhlee@wow.hongik.ac.kr,schultz@umich.edu and \\ ceccio@umich.edu
}

Received 29 August 2002, in final form 26 February 2003

Published 22 July 2003

Online at stacks.iop.org/IP/19/919

\begin{abstract}
A singularity image method is applied to the electrical impedance tomography of gas-liquid flows in a two-dimensional circular domain. Algorithms that use analytic complex functions, dipoles and the Milne-Thomson circle theorem are described. Numerical experiments are provided to demonstrate the robustness of this technique. Numerical results show excellent reconstruction properties.

(Some figures in this article are in colour only in the electronic version)
\end{abstract}

\section{Introduction}

Electrical impedance tomography (EIT) is a noninvasive imaging technique that determines the impedance distribution of a complex medium inside a body by measuring voltage or current on the body's surface. EIT is an inexpensive alternative to other methods for reconstruction of complex materials, such as magnetic resonance imaging (MRI), x-ray computed tomography (CT) and other imaging methods. It has become a focus of many investigators engaged in medical and process imaging and other related fields. There have been numerous publications on this issue and extensive reviews can be found in survey articles (Barber and Brown 1984, Ceccio and George 1996, Ramm 1998 and Cheney et al 1999).

EIT is a well-known inverse problem, with several developed reconstruction methods. Popular algorithms used are the back-projection method (Barber and Brown 1992) and an iterative procedure where a finite-element method solves the forward problem (Yorkey 1990, Jain et al 1997, Vauhkonen et al 1998). Boundary element methods are also employed when the conductivity distribution is composed of two different constant and uniform electrical properties (Lemonnier and Peytraud 1998).

The electrical potential $\phi$ in the domain is governed by the generalized Laplace equation

$$
\nabla \cdot \sigma \nabla \phi=0
$$




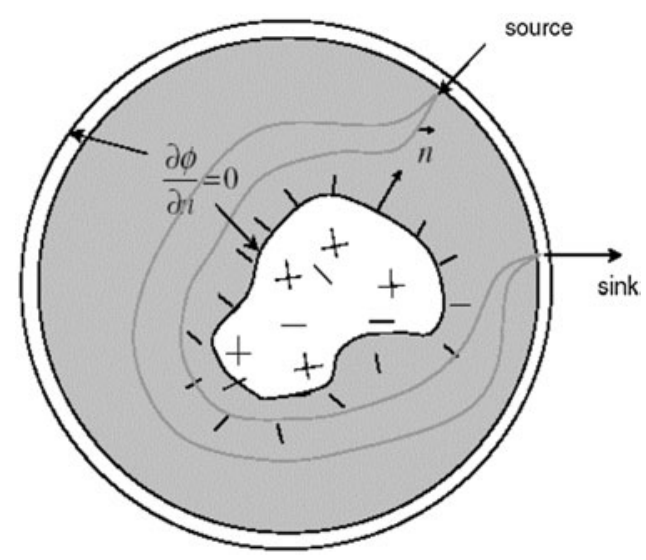

Figure 1. Schematic of one arbitrary shape bubble in the domain. The grey region represents the conducting (liquid region). The differential equation is exactly satisfied by the $M$ singularities (represented by + and - in this figure) outside the computational (conducting liquid) domain. The Neumann boundary conditions on the cylinder wall $(R=1)$ are also identically satisfied by images, except for the singularities at the point source and sink electrodes. The void (bubble) Neumann conditions are approximated by the dipole singularities. The iterative inverse procedure reconstructs an approximation of the void(s) shape for examining voltages at the pipe wall for several source-sink combinations of locations.

with the boundary condition

$$
\vec{n} \cdot \sigma \nabla \phi=I .
$$

Here $\sigma$ is the electrical conductivity (or inverse impedance), $\vec{n}$ is the outward norm on the boundary, and $I$ is the applied current flux on the domain boundary. For this study, the electrodes are infinitesimal and so the right-hand side of (2) is a sum of two Dirac $\delta$ functions of the opposite sign with singularities at the two electrode locations as shown in figure 1.

In general and depending on the frequency of excitation, $\sigma$ is complex, with the real and imaginary parts respectively representing the resistive and reactive components. Then $\phi$ is complex as well. For the purposes of this study, we will assume that $\sigma$ is purely resistive or reactive (capacitive) and then $\phi$ is real. Otherwise, the real and imaginary parts of $\phi$ can be handled separately as only linear problems are considered here.

The impedance may be considered binary when a constant-impedance fluid is distributed uniformly over the medium except at some inclusions where the electrical conductivity virtually vanishes. The imaging of bones, gas-liquid flows, and buried insulated cables may serve as good examples. We will consider only this case here.

The application of a two-dimensional electrical impedance tomographic system to the imaging of gas-liquid flows in a circular domain of radius $R$ is considered in this study. Also in this case, the electrical impedance of the two phases are significantly different; the component having the higher resistance, as is often the case of a gaseous phase, can be considered a perfect electric insulator. A linear potential combined with a dipole represents a current flow about a cylinder. In this study, a singularity image method (SIM), where the electric potential and the current flow around the non-conductive phase is represented by singularities, is developed to identify the infinite impedance of the gas of two-phase flows. We will assume that the gas phase void fraction is small and all of the electrodes are 'wet' with the higher conductivity phase.

Obviously, bubbles (or voids) are more spherical than cylindrical, and hence the electrical field will be three-dimensional. If the electrodes are long and the axial variations in the field 
impedance are small, the axially averaged electric field is representative of the impedance distribution in the two remaining dimensions. In converting this to a void fraction, it is important to use the 3D version of the Maxwell-Hewitt relation for interpreting experimental data from nominally spherical voids rather than the $2 \mathrm{D}$ version that we use here for the analytical interpretation of cylindrical voids. If not, a three-dimensional point singularity is required. Then the complex analytical function method and the circle theorem are no longer valid. Although we have 'designed' three-dimensional singularities with images that approximately satisfy the Neumann boundary condition, the increased computational effort has delayed implementation until future study.

Like all inverse problems, this method is strongly dependent on data noise. For the purposes of this first study, we will only consider numerical experiments essentially free of noise. In section 2 we show how the forward problem can be described by an analytical representation that is composed of a relatively small number of complex singularities. Section 3 describes the inverse procedure. Section 4 shows several numerical examples.

\section{Forward problem in 2D two-phase flow and circle theorem}

In the liquid region of the gas-liquid flows the electrical conductivity $\sigma$ is assumed constant, so the electrical potential $\phi$ satisfies the Laplace equation

$$
\nabla^{2} \phi=0 \text {. }
$$

For two-dimensional $(x, y)$ potential problems, it is convenient to define a complex function $w(x+\mathrm{i} y)=w(z)=\phi(z)+\mathrm{i} \psi(z)$ such that $w$ is an analytic function and the current stream function $\psi$ also satisfies

$$
\nabla^{2} \psi=0
$$

On the gas-liquid phase interfaces as well as the circular domain boundary $|z|=R$ (the coordinate system is placed on the circle centre), the following boundary conditions should be satisfied:

$$
\frac{\partial \phi}{\partial n}=\psi-\text { constant }=0
$$

In practice, a set of electrodes is placed on the perimeter of the domain in an EIT system. Two of them are typically selected temporarily as a current source and sink. The twodimensional complex potential field formed by a point source-sink pair of the same strength in free space is

$$
w(z)=\frac{q}{2 \pi}\left\{-\ln \left(z-z_{+}\right)+\ln \left(z-z_{-}\right)\right\},
$$

where $q$ is the known source strength and $z_{+}$and $z_{-}$are the locations of the source and sink, respectively. There are some practical problems associated with small electrodes, but for simplicity we will assume infinitesimal electrodes here. This requires that the source, sink and measurement locations are mutually exclusive since voltages are singular at the source and sink. To satisfy the Neumann boundary conditions on the outer boundary, the Milne-Thomson (1960) circle theorem is applied. The base potential field without any bubble in the domain and on the boundary is then

$w_{1}(z)=\frac{q}{2 \pi}\left\{-\ln \left(z-z_{+}\right)-\ln \left(R^{2} / z-\bar{z}_{+}\right)+\ln \left(z-z_{-}\right)+\ln \left(R^{2} / z-\bar{z}_{-}\right)\right\}$,

where the bar denotes the complex conjugate. The second and fourth terms on the right-hand side of (7) represent the image singularities that satisfy the outer Neumann boundary condition. For simplicity, the domain radius $R, q / 2 \pi$ and $\sigma$ are normalized to 1 in what follows. 
A bubble produces a perturbation to the base potential field. The complex potential due to a dipole centred at $z_{\mathrm{d}}$ in free space is given by

$$
w(z)=\frac{a}{z-z_{\mathrm{d}}},
$$

where $a$ is the strength of the dipole that can be calculated by

$$
a=r^{2} S \mathrm{e}^{-\mathrm{i} \theta},
$$

where $r$ is the bubble radius when $S$ and $\theta$ are the magnitude and the phase of the gradient of electrical potential at the bubble centre without the dipole. Since the bubble is not in a perfectly local uniform flow, the bubble boundary will not be perfectly circular. The adjustment of (8) to satisfy the boundary condition (5) on the bubble surface and the domain boundary at $|z|=R$ can be accomplished by applying the circle theorem as follows:

$$
w(z)=\frac{a}{z-z_{\mathrm{d}}}+\frac{\bar{a}}{1 / z-\bar{z}_{\mathrm{d}}}
$$

where the second term on the right-hand side is the image singularity of the dipole. When there are sparsely distributed $M$ bubbles in the medium, and if each bubble is located far enough from the others, each bubble can be replaced by a dipole so that the perturbation that meets (5) is given by

$$
w_{2}(z)=\sum_{m=1}^{M}\left(\frac{a_{m}}{z-z_{\mathrm{d}}}+\frac{\bar{a}_{m}}{1 / z-\bar{z}_{\mathrm{d}}}\right) .
$$

The strength of dipole $a_{m}$ is computed from the electrical potential field formed by the current source, sink, all other dipoles and their image singularities, which produces locally uniform current flow at $z_{\mathrm{d} m}=x_{\mathrm{d} m}+\mathrm{i} y_{\mathrm{d} m}$. The $a_{m}$ are computed in an iterative way because the strength of each dipole affects the others. The complex electrical potential which includes current source, sink and $M$ dipoles is obtained by the superposition of $w_{1}(z)$ and $w_{2}(z)$ :

$$
\begin{gathered}
w_{3}(z)=\left[-\ln \left(z-z_{+}\right)-\ln \left(\frac{1}{z}-\bar{z}_{+}\right)+\ln \left(z-z_{-}\right)+\ln \left(\frac{1}{z}-\bar{z}_{-}\right)\right] \\
+\sum_{m=1}^{M}\left(\frac{a_{m}}{z-z_{\mathrm{d}}}+\frac{\bar{a}_{m}}{R^{2} / z-\bar{z}_{\mathrm{d}}}\right) .
\end{gathered}
$$

If the bubble spacing to diameter ratio becomes too low, the free surface formed by a dipole deviates significantly from a circle. Solving the forward problem requires additional singularities to form a circle. On the boundary of a bubble, there exist two stagnation points. At these points, $w$ is required to satisfy the following condition:

$$
\frac{\mathrm{d} w}{\mathrm{~d} z}=0
$$

Since $\mathrm{d} w / \mathrm{d} z$ can be approximated by

$$
\frac{\mathrm{d} w}{\mathrm{~d} z} \approx\left(\frac{\mathrm{d} w}{\mathrm{~d} z}\right)_{0}+\left(\frac{\mathrm{d}^{2} w}{\mathrm{~d} z^{2}}\right)_{0}\left(z-z_{0}\right)
$$

at $z_{0}$, the vicinity of a stagnation point, the locations that satisfy $\mathrm{d} w / \mathrm{d} z=0$ can be found by

$$
z=z_{0}-\frac{(\mathrm{d} w / \mathrm{d} z)_{0}}{\left(\mathrm{~d}^{2} w / \mathrm{d} z^{2}\right)_{0}}
$$

Starting from an initial guess of $z_{0}$, the first and second derivatives of $w$ can be computed from (12) and $z$ is updated by (15). A Newton-Raphson procedure iterates until $\left|(\mathrm{d} w / \mathrm{d} z)_{0}\right|$ becomes negligibly small to obtain the stagnation point. The bubble shape is determined by a line of constant $\psi$ connecting the stagnation points. The bubble shape will differ slightly for different current source and sink combination, as will the stagnation points. 


\section{Inverse problem}

Let $\Delta \phi_{j k}$ be the measurement of the electrical potential perturbation at the $k$ th electrode for some $j$ current source-sink combination index caused by the presence of bubbles in the domain. The estimated electrical potential difference $\Delta \phi_{j k}$ can be defined as (with $R$ made dimensionless to 1 )

$\Delta \phi_{j k}=\operatorname{Re}\left[w_{3}\left(z_{k}\right)_{j}-w_{1}\left(z_{k}\right)_{j}\right]=\sum_{m=1}^{M} \operatorname{Re}\left[\left(\frac{a_{m}}{z_{k}-z_{\mathrm{d} m}}+\frac{\bar{a}_{m}}{1 / z_{k}-\bar{z}_{\mathrm{d} m}}\right)_{j}\right]$.

The residual $F_{j k}$ is defined by comparing the measured potential difference that is estimated by a trial singularity distribution:

$$
F_{j k}=\Delta \phi_{j k}-\Delta \Phi_{j k}
$$

When the total number of electrodes along the domain boundary is $N$ and the source and sink electrodes are chosen, $(N-2)$ data points are obtained from the other electrodes. For $N_{\mathrm{s}}$ combinations of source and sink location, the number of independent measurements and hence equations in the inverse problem is

$$
N_{\mathrm{e}}=(N-2) N_{\mathrm{s}} \text {. }
$$

An iterative procedure determines the location and size of each singularity. The location of the singularity does not shift as the bubbles are assumed to be stationary for all measurements during all electrode pattern combinations. However, the strength $a_{m}$ of each dipole varies as the source and sink locations shift. Therefore, the total number of real unknowns for the problem with $M$ dipoles is

$$
N_{\mathrm{u}}=2 M\left(1+N_{\mathrm{s}}\right)
$$

where $2 M$ comes from the unknown dipole locations and $2 M N_{\mathrm{s}}$ comes from the complex dipole strength that must be found for each of the $N_{\mathrm{s}}$ combinations of source and sink location. The determination of the maximum number of $N_{\mathrm{s}}$ for a given $N$ is a subtle issue depending on the ability to perform superposition. If the electrodes prescribe voltage instead of current or if the electrode is of finite size, superposition is not possible and the total possible combinations is given by

$$
N_{\mathrm{s}}=\left(\begin{array}{c}
N \\
2
\end{array}\right)=N(N-1) / 2
$$

as stated in Ceccio and George (1996). Finally, the current pattern for two similar independent patterns would have to be subtracted from each other for other combination flows and this could be fraught with error if noise were significant. However, for the analytical examples in our analysis, the number of combinations required is $N-1$.

Even without the introduction of noise, it is important that the systems be sufficiently overdetermined. We find that $N_{\mathrm{e}}$ should be typically twice that of $N_{\mathrm{u}}$ for good convergence properties in the iterative inverse procedure as well as for spatial resolution of the void profiles with increased $N$.

\section{Numerical examples}

For all the following examples, 24 evenly spaced electrodes are located on the boundary of a circular domain with unit radius. The electrodes at $\theta=0, \pi / 2, \pi$ and $3 \pi / 2$ serve as current sources or sinks, thus making the number of independent source and sink combinations $N_{\mathrm{s}}$ to be 3, although for the purpose of illustrating our examples here we consider the more general case of $N_{\mathrm{s}}=N(N-1) / 2$ as described above. 


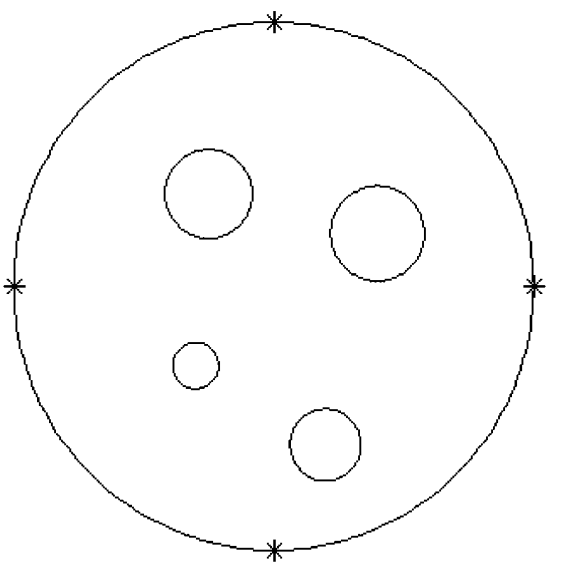

Figure 2. Bubble locations and sizes for example in the $2 \mathrm{D}$ domain $(*$ denotes source/sink locations).



Figure 3. Estimated potential difference along the boundary for six source/sink pair combinations (see figure 8 for symbol legend). The symbols are placed at the electrode locations.

\section{Few bubbles}

With SIM in mind, several situations may be considered: (i) each dipole representing a bubble, (ii) a dipole representing many bubbles and (iii) several dipoles representing a bubble. This study concentrates on the first example although preliminary work reported here shows that the other two cases are also well represented by SIM. This means that the number of bubbles does not need to be known a priori.

An example case with four bubbles placed in the domain is shown in figure 2. First, SIM is applied to solve the forward problem and produce the 'data' for the inversion algorithm. Figure 3 shows the estimated $\Delta \phi_{j k}$ for each current source and sink combination $j$ that are then input as the measurement data $\Delta \phi_{j k}$ for the inverse problem. While the forward problem is solved for four bubbles, the inverse problem is solved for several values of $M$ for the purpose of comparison. 

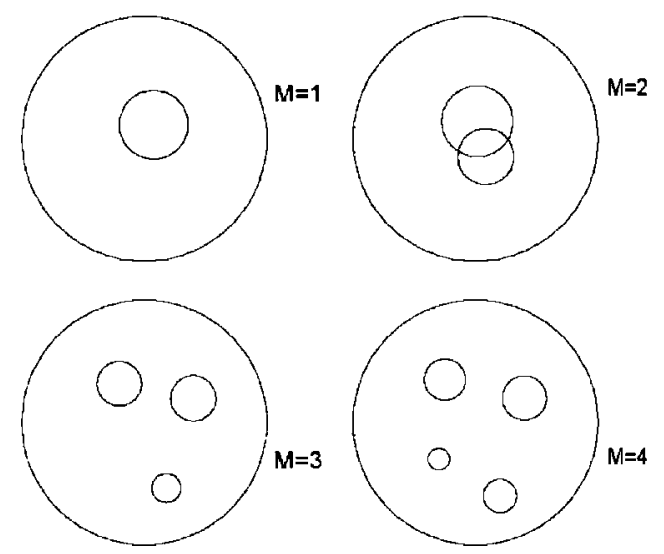

Figure 4. Locations and sizes of the estimated circular voids as a function of the number of dipoles used in the computation, $M$.

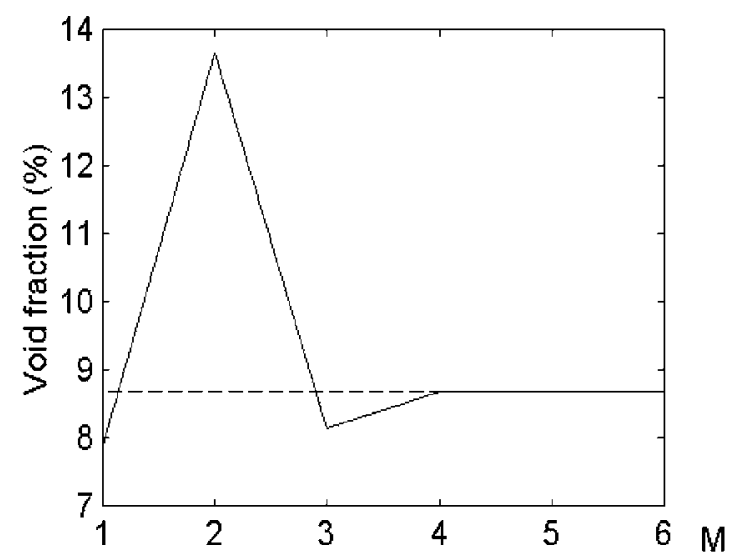

Figure 5. Estimated void fraction as a function of the number of singularities $M$ used in the computation. Dotted line represents the exact void fraction. The large error for $M=2$ comes from overlap of the two bubbles (see figure 7).

Figure 4 shows the estimated locations and sizes of bubbles for each assumed $M$ as the result of the inverse problem when the bubble size is determined from (9). Figure 5 shows the resulting void fractions for each value of $M$. The dotted line indicates the actual void fraction of this example. The computed void fraction is only $0.42 \%$ lower than the actual void fraction when one dipole $(M=1)$ is used to simulate the four bubbles; however, $M=2$ gives a bigger discrepancy. It is readily seen in the figure 5 that the estimated locations and sizes of bubbles converges to those prescribed as $M$ increases and that the overlap of bubbles for the simplified procedure in figure 4 for $M=2$ is responsible for the error in the calculation of void fraction. For $M \geqslant 4$, the void fraction looks almost identical to the original void fraction, indicating that the locations and sizes of bubbles are estimated accurately. However, the convergence of the iterative process may not be achieved for higher $M$ since the system with $N_{\mathrm{s}}=3$ does not become sufficiently overdetermined. Figure 6 shows the reduction of maximum residual as $M$ increases, and figure 7 shows the estimated potential difference $\Delta \phi_{j k}$ along the boundary as $M$ varies when the source and sink are located at $\theta=\pi$ and $3 \pi / 2$, respectively. This reveals that 


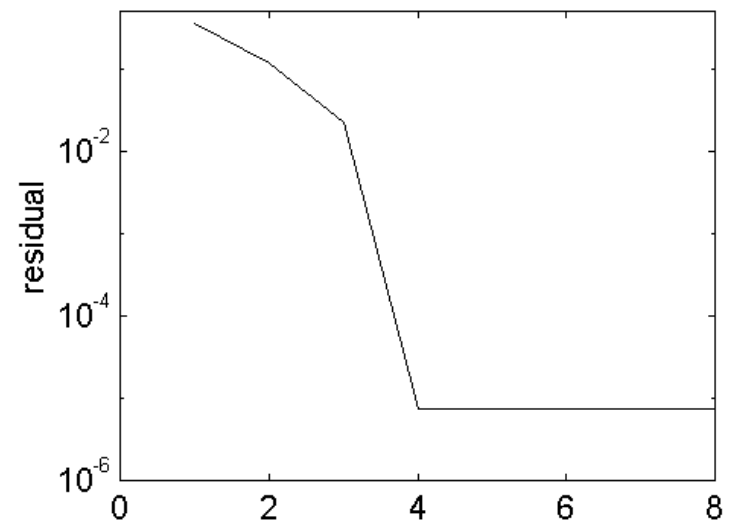

Figure 6. Residual as a function of the number of singularities $M$ used in the computation.
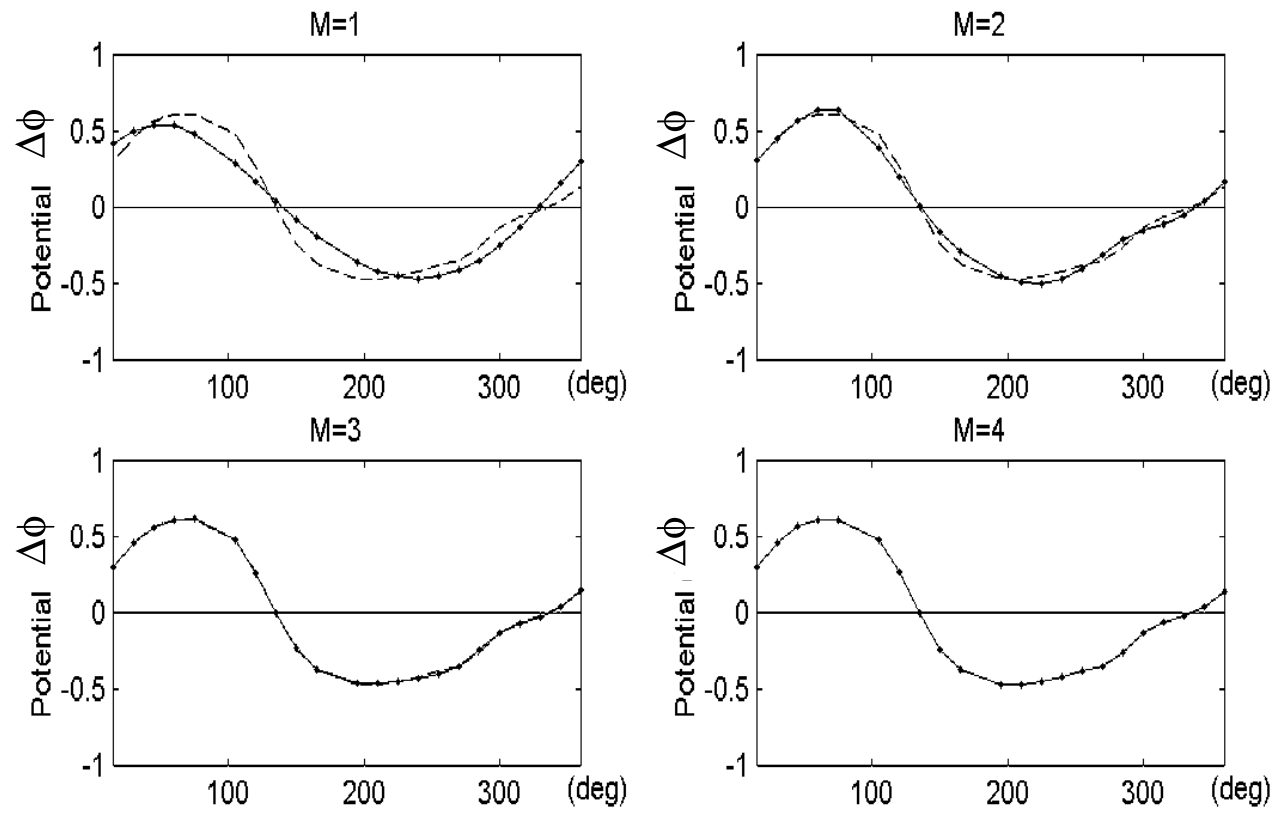

Figure 7. Estimated potential disturbances for the inverse problem as the number of dipoles varies The dashed curve represents the exact solution of the forward problem for one of the source-sink pairs shown in figure 3 (with solid dots). The source and sink are located at $\pi / 2$ and $\pi$, respectively for this example.

the estimated locations and shapes of bubbles are found to be identical with input for $M \geqslant 4$. Figure 8 depicts the electrical potential as well as the current flow for each current source and sink combination when the number of dipoles, and hence the estimated number of bubbles, $M$ is set to 4 . The bubble shape, determined by the dividing current line, is exact and nearly circular. Although tedious, the void fraction for this example with $M=2$ would give a good result if the dividing current lines were used instead of the method assuming circular voids because the bubbles would be distorted rather than overlapping. 


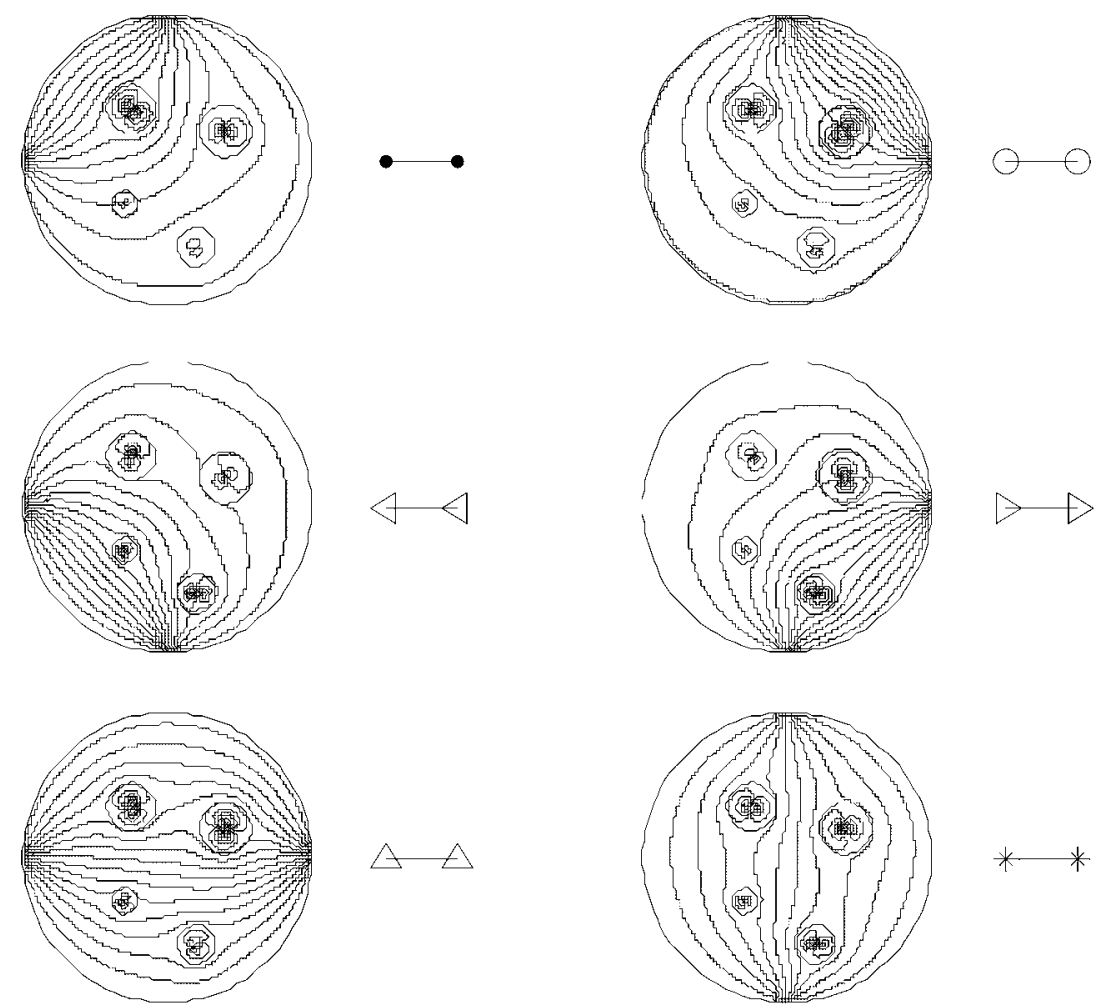

Figure 8. Reconstruction of four bubbles using four dipoles for various source/sink pairs. Lines represent electrical current lines. Symbols next to each diagram match those of figure 3 .

\section{Many bubbles}

That the example above gives a very good measure of the void fraction even when one singularity is used to represent four bubbles, is a good indication that many bubbles may be represented by relatively few singularities. This is especially important since most flows of interest will have a large number of bubbles (voids) in the domain. Often, obtaining the void fraction or other low-order moments is the main goal. In that case, it is not necessary to accurately know the distribution of individual bubbles. In this second example, we use only a few dipoles to represent 20 small bubbles in the domain for the same source sink combination as above. The bubble shapes and locations are shown in figure 9, which also shows the equipotential and current lines for one source-sink combination for the forward problem. Only a few dipoles $(M \ll 20)$ are used to simulate this case, and the result is shown in figure 10 as the void fraction. The maximum error (approximately $11 \%$ ) occurs when $M=2$. But when $M$ is equal to or larger than 4, the error is less than $3 \%$. Figure 11 shows the maximum residual. These results show that this method can be used to measure void fraction for practical flows.

\section{Non-circular bubbles}

To test the capability of this technique for obstacles of arbitrary shape, an elliptical bubble example is given. The forward problem is constructed using 20 dipoles inside the bubble to satisfy the boundary conditions at 300 locations on the bubble. Unlike the previous examples, 


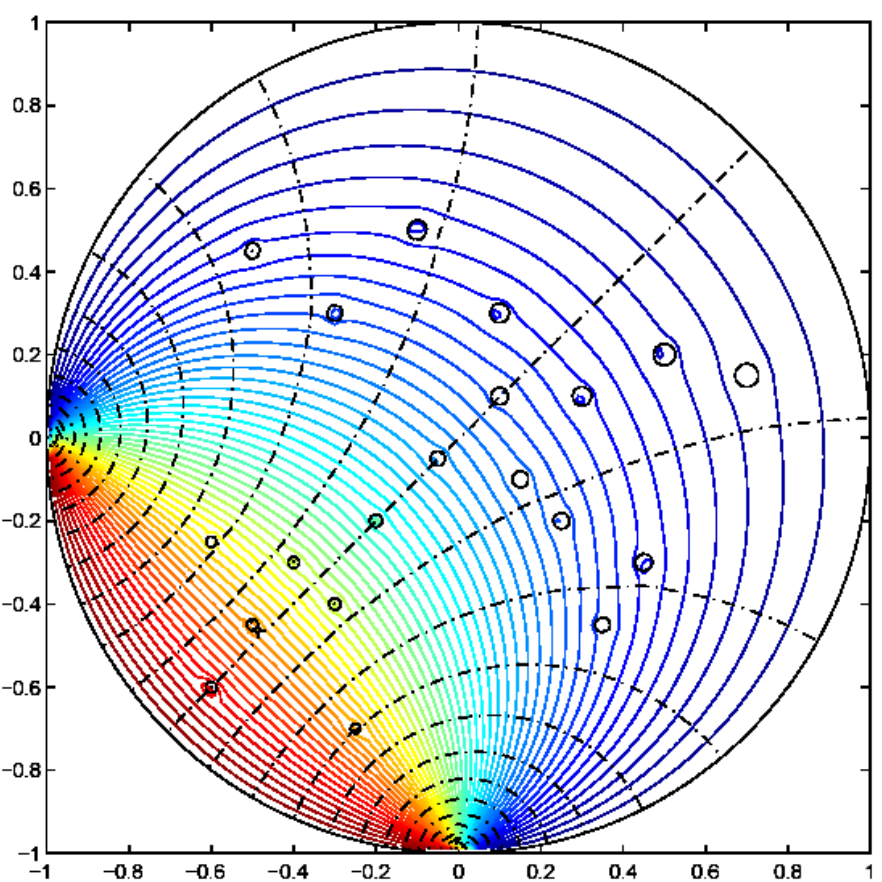

Figure 9. Equipotential and current lines for an example with $N=20$ bubbles of much smaller diameter. The circles shown are placed at their known locations while the computational lines are shown for one particular source/sink pair from the forward problem.

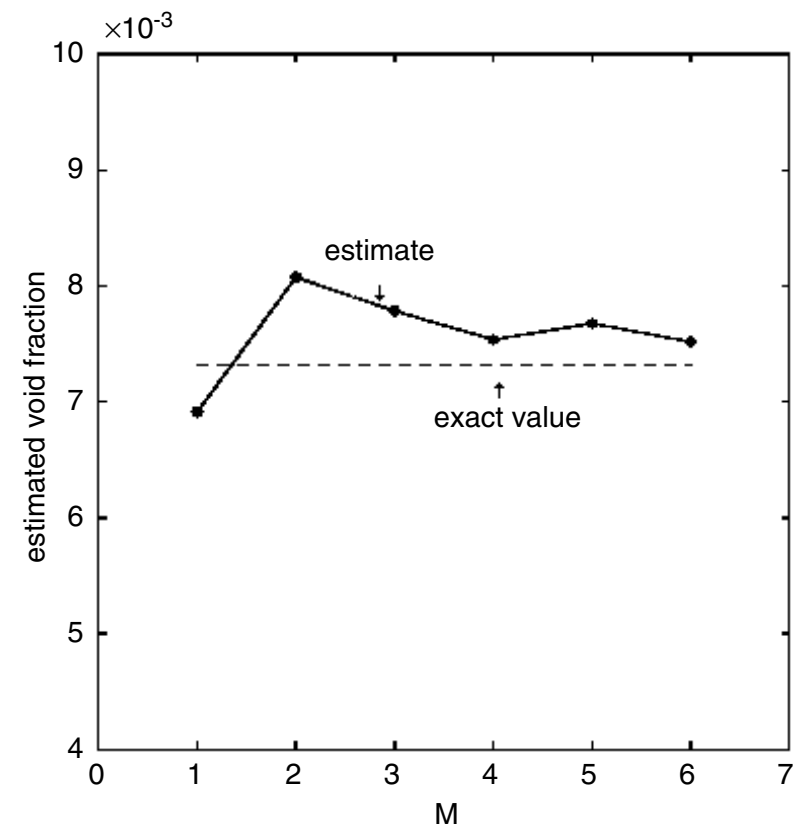

Figure 10. Estimated void fraction for the example of figure 9 for varying number of dipoles, $M$. The error is less than $0.2 \times 10^{-3}$ for $M=6$. 


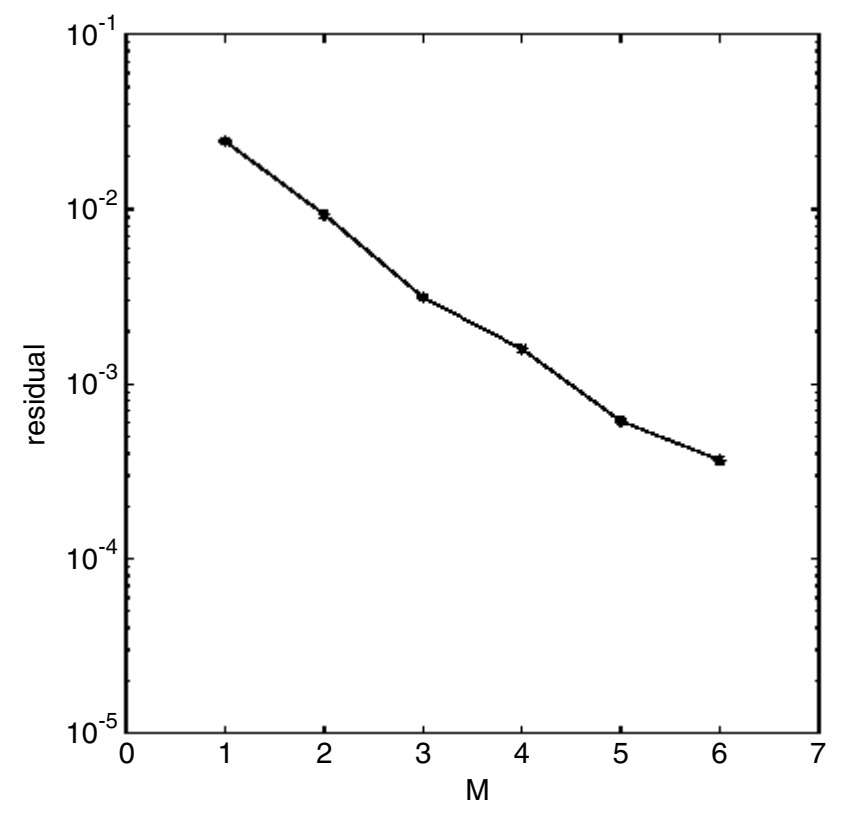

Figure 11. The residual for the example of figure 9 is shown for varying number of dipoles, $M$. The residual is exponentially decreasing with $M$.

this requires the solution of a well overdetermined linear algebraic system to create the forward solution. These solutions for various source/sink pairs produce the 'experimental' voltage measurements on the insulated wall boundary to calculate the inverse problem as shown in figure 12. A varying number of dipoles $M$ is chosen to perform the inverse problem. For one dipole $(M=1)$, the solution expresses approximately a circle. When number of dipoles is larger than one, the solutions approach the shape and location given with increasing of the number of dipoles. When $M=3$, the calculated shape is well within $1 \%$ error (not shown). Figure 13 shows the residual of the inverse problem. A square void was also considered but is not shown. Aside from the inevitable rounding in the corner vicinity and the larger number of singularities required for good reconstruction, the singularity method performed well. These results show that this technique can be used to estimate a volume fraction and reconstruct arbitrary shape bubbles in gas-liquid flows, and it also can be used to reconstruct other materials whose conductivities are significantly different.

\section{Conclusion}

The SIM is excellent for two-dimensional EIT where the electrical conductivity is uniformly distributed over a circular domain except at some inclusions where the conductivity vanishes. The Milne-Thomson circle theorem satisfies the conditions on the domain boundary, and the regions with higher impedance are represented by dipoles. Algorithms for the forward and the inverse problems are developed. Examples show that the present method is applicable to the estimation of the void fraction in bubbly flows. The method is also useful in the reconstruction of interfaces where impedances of the components are significantly different.

Preliminary numerical tests show that noise has no more than the expected degradation of the accuracy of the method. We also have developed a 3D singularity method that approximately satisfies the Neumann condition on the cylindrical boundary. Considerably 

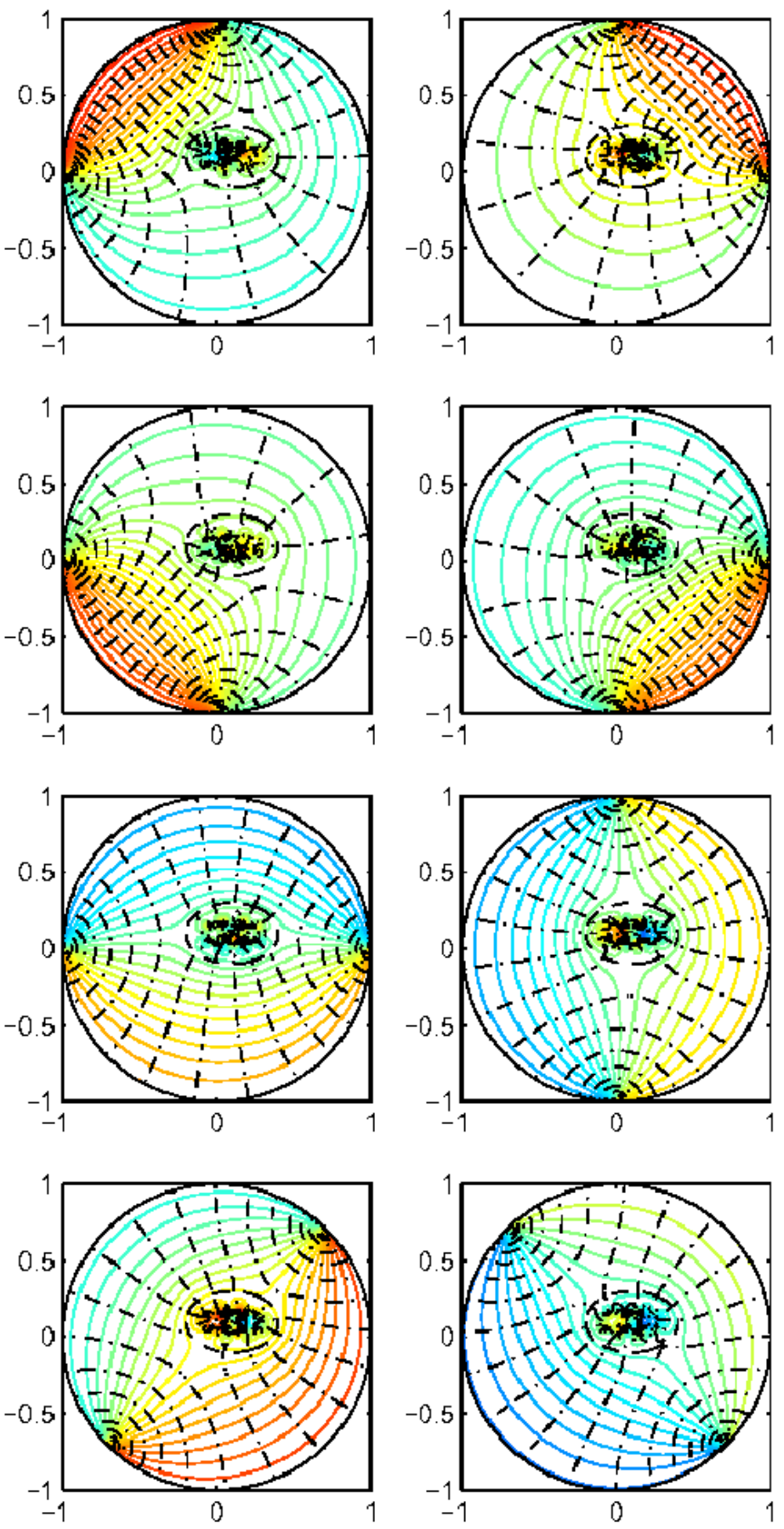

Figure 12. The forward solutions for the elliptical example is shown for all source/sink combinations. The solution inside the ellipse is now quite complex because of the large number of sources and sinks. For this case, two extra source/sink combinations (at the bottom of this figure) were added for improved accuracy. 


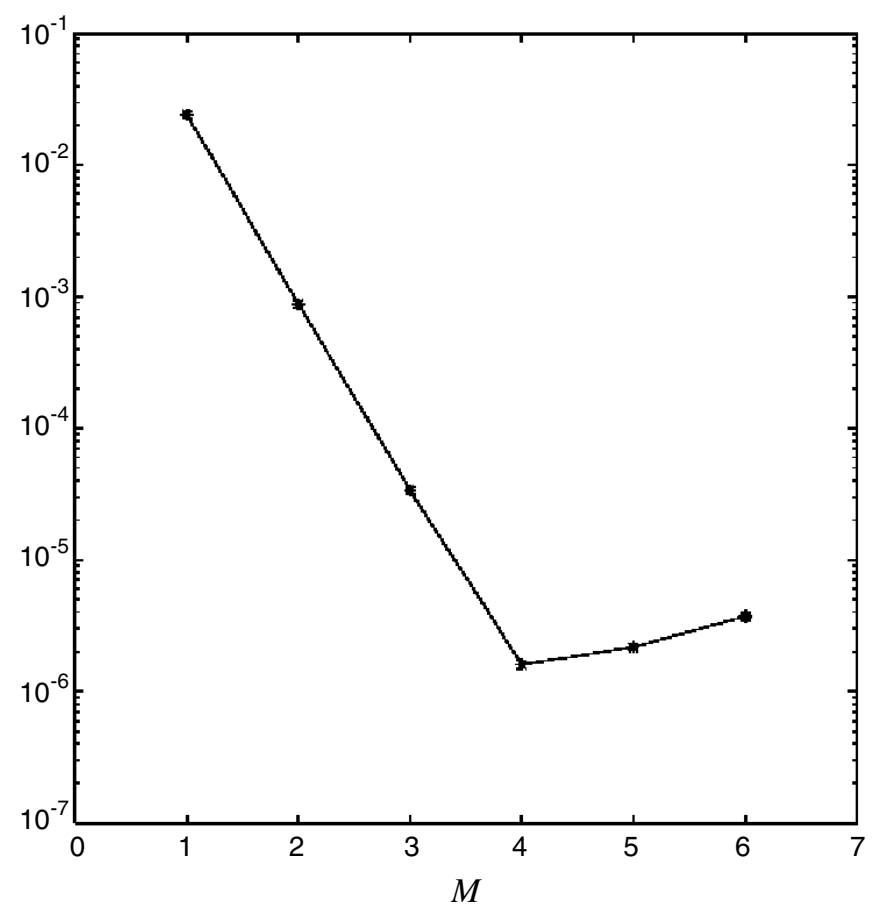

Figure 13. The residual decreases exponentially for the elliptical void example until $M=4$ and precision difficulties occur.

more effort is required and so 2D reconstruction is preferred when appropriate. Finally, for 2D reconstruction, non-cylindrical containers can be considered using conformal mapping. We postpone discussion of all these efforts to a future paper.

\section{Acknowledgment}

This work is supported by the National Science Foundation under grant no 0074245.

\section{References}

Barber D C and Brown B H 1984 Applied potential tomography J. Phys. E: Sci. Instrum. 17 723-33

Barber D C and Brown B H 1992 Image reconstruction in electrical impedance tomography SPIE Mathematical Methods in Medical Imaging vol 1768 (Bellingham, WA: SPIE Optical Engineering Press) pp 60-8

Ceccio S L and George D L 1996 A review of electrical impedance and techniques for the measurement of multiphase flows J. Fluids Eng. 118 391-9

Cheney M, Isaacson D and Newell J C 1999 Electrical impedance tomography SIAM Rev. 41 85-101

Jain H, Isaacson D, Edic P M and Newell J C 1997 Electrical impedance tomography of complex conductivity distributions with noncircular boundary IEEE Trans. Biomed. Eng. 44 1051-60

Lemonnier H and Peytraud J F 1998 Is 2D impedance tomography a reliable technique for two-phase flow? Nucl. Eng. Des. $184253-68$

Milne-Thomson L M 1960 Theoretical Hydrodynamics (London: Macmillan)

Ramm A G 1998 Inverse Problems, Tomography, and Image Processing (New York: Plenum)

Vauhkonen M, Karjalainen P A and Kaipio J P 1998 A Kalman filter application to track fast impedance changes in electrical impedance tomography IEEE Trans. Biomed. Eng. 45 486-93

Yorkey T J 1990 Electrical impedance tomography with piecewise polynomial conductivities J. Comput. Phys. 94 344-60 